\title{
Nivel de conocimiento y conducta de docentes de educación escolar básica de escuelas públicas frente a casos de avulsión y fractura dentaria
}

\section{Resumen}

Objetivo: Evaluar el nivel de conocimiento y conducta de los docentes de Educación Inicial, docentes del 1er Ciclo, docentes del 2do Ciclo y docentes de Educación Física de las escuelas públicas de la ciudad de San Lorenzo (Paraguay) frente a casos de Avulsión y Fractura Dentaria durante el año 2009. Material y Método: El diseño fue tipo observacional descriptivo de corte transversal. El muestreo fue probabilístico; teniendo en cuenta los criterios de selección dentro de una muestra de 141 docentes. Se clasificaron los datos según el género, grupo etáreo, grado de capacitación, y años de experiencia profesional; para lo cual se confeccionó un cuestionario. Resultados: El nivel de conocimiento sobre avulsión y fractura dentaria más frecuente de los docentes fue el moderado con un $50 \%$ (70/141), seguido por el conocimiento bajo con un $27 \%$
(38/141). La conducta a seguir sobre avulsión y fractura dentaria más frecuente por los docentes fue el regular con un $47 \%(66 / 141)$, seguido por la conducta a seguir favorable con un $30 \%$ (42/141). Conclusión: el nivel de conocimiento sobre avulsión y fractura dentaria más frecuente fue el moderado, en cuanto a los resultados de la conducta a seguir el más frecuente fue el de regular. Teniendo en cuenta los datos anteriormente presentados se torna necesario implementar campañas educativas sobre la atención de emergencias en Traumatismos Dentales en las escuelas, ya que en el ambiente escolar la persona más cercana al niño es el docente y su intervención en el momento que el niño sufre el traumatismo dental puede ser determinante para la resolución de los daños que éste pueda haber sufrido.

Palabras Claves: Traumatismos de los dientes, Avulsión dentaria, Fractura dental.

Artigo Original

\section{Nível de conhecimento e conduta dos docentes de educação básica de escolas públicas frente a casos de avulsão e fratura dentária}

\section{Resumo}

Objetivo: Avaliar o nível de conhecimento e conduta dos docentes de educação básica, do- centes do $1^{\circ}$ Ciclo, docentes do $2^{\circ}$ Ciclo e docentes de Educação Física das escolas públicas da cidade de San Lorenzo (Paraguai) frente a casos de avulsão e fratura dentária durante o ano

\footnotetext{
${ }^{1}$ Especialista en Odontopediatria de la Facultad de Odontología Universidad Autónoma del Paraguay (UAP).

${ }^{2}$ Especialista y Máster en Odontopediatria de la Facultad de Odontología Universidad Autónoma del Paraguay (UAP).

${ }^{3}$ Coordinadora de Posgrado de Odontopediatria de la Facultad de Odontología Universidad Autónoma del Paraguay (UAP).
} 
2009. Material e Métodos: O desenho foi tipo observacional descritivo de corte transversal. A amostragem foi probabilística; considerando os critérios de seleção dentro de uma amostra de 141. Os dados foram classificados segundo o gênero, grupo etário, grau de capacitação, e anos de experiência profissional; para o que se elaborou um questionário. Resultados: $\mathrm{O}$ nível de conhecimento sobre avulsão e fratura dentária mais frequente foi o moderado em $50 \%$ (70/141) dos docentes, seguido pelo baixo em $27 \%$ (38/141). A conduta para os casos de avulsão e fratura dentária mais frequente foi a regular em $47 \%$ (66/141) dos docentes, seguido pela conduta favorável em 30\% (42/141). Conclusão: o nível de conhecimento sobre avulsão e fratura dentária mais frequente foi o moderado e com relação à conduta o mais frequente foi a regular. Considerando os dados anteriormente apresentados torna-se necessário implementar campanhas educativas sobre a atenção de emergências de traumatismos dentários nas escolas, já que no ambiente escolar a pessoa mais próxima à criança é o professor e sua intervenção no momento que a criança sofre o traumatismo dentário pode ser determinante para a resolução das injúrias sofridas.

Palavras-chave: Traumatismos dentários, Avulsão dentária, Fraturas dos dentes.

Original article

\section{Knowledge and behavior level of basic education teachers in public schools deal with cases of tooth avulsion fracture}

\section{Summary}

Objective: Assess the level of knowledge and behavior of early education teachers, teachers of the 1st cycle, 2nd cycle teachers and physical education teachers in the public schools of the city of San Lorenzo (Paraguay) versus cases Tooth avulsion and fracture during the year 2009. Material and Methods: The design was an observational cross-sectional descriptive. Sampling was probabilistic, taking into account the selection criteria within a sample of 141 teachers. Data were classified according to gender, age group, level of training, and years of professional experience, for which a questionnaire was made. Results: The level of knowledge about tooth avulsion fracture more frequently teachers was moderate with $50 \%$ (70/141), followed by low knowledge with $27 \%$ (38/141). he action to take on tooth avulsion fracture most often used by the regular teachers was $47 \%(66 / 141)$, followed by the action to take favorable with $30 \%(42 / 141)$. Conclusion: The level of knowledge about tooth avulsion fracture was the most frequent moderate results in terms of behavior to follow the most common was to regulate. Considering the above data it becomes necessary to implement educational campaigns about emergency care in Dental Trauma in schools, because in the school environment the person closest to the child's teacher and his speech at the time that the child has dental trauma can be decisive for the resolution of the damage it may have suffered.

Keywords: Injuries of the teeth, tooth avulsion, tooth fractures. 


\section{Introducción}

Los Traumatismos Dentales son lesiones de los dientes o tejidos blandos producidas por una acción violenta sobre la cavidad bucal, pudiendo ocasionar la fractura o pérdida de uno o varios dientes. Aunque hoy en día los traumatismos dentales son la segunda causa de atención odontopediátrica tras la caries, en un futuro muy cercano la incidencia de las lesiones traumáticas constituirá la principal demanda de atención. (1-3)

Varios autores han realizado clasificaciones sencillas de los traumatismos, actualmente es casi universal el uso de la clasificación de Andreassen, que es una modificación de la propuesta por la organización mundial de la salud aceptada internacionalmente y se basa en las diferentes pautas de tratamiento según la patología traumática; que consiste en, lesiones de tejidos dentarios duros y de la pulpa, lesiones de los tejidos periodontales, lesiones del hueso de soporte, lesiones de la mucosa oral y de la encía (1-14).

La edad es un factor importante en los traumatismos dentales los picos de máxima frecuencia en la dentición permanente se encuentra entre los nueve y diez años. La prevalecía en la dentición temporal varía entre los diez y veinticuatro meses. $(1-3 ; 5,7,8,10)$

En la dentición permanente los varones sufren, estadísticamente, más lesiones que las mujeres siendo algunas de las causas caídas, golpes, accidentes ciclísticos, prácticas deportivas, maltrato infantil etc. $(1-3 ; 4,5,7,9,10)$ La diferencia por genero en la dentición temporal no es tan evidente ya que generalmente su causa se debe a los intentos del niño por caminar, a la falta de coordinación, choque con mesas bajas y por no total desarrollo del control motor y el equilibrio. $(1-3 ; 4,7,10)$
Para la dentición permanente la mayoría de los estudios revelan que la lesión más frecuente es la fractura de la corona no complicada siendo el diente más afectado el incisivo central superior seguido por el lateral superior e incisivo central inferior. El ángulo mesioincisal es el que se fractura con más frecuencia. (1-3; 5,7-10). En la dentición primaria la lesión más frecuente es la luxación. $(2,3,8,10)$

Se han realizado varios estudios en diferentes países para conocer el nivel de información de las personas sobre el traumatismo dentario en la gran mayoría de ellos se llego a la conclusión que esa información es muy escasa.

En un trabajo realizado en el año 2005 aplicaron un cuestionario a 78 profesores en Porto y Estambul, 23 habían tenido previamente educación formal del trauma dental. De los profesores entrevistados, 58 de ellos admitieron no tener ningún conocimiento del trauma dental. Referente al conocimiento, 29 profesores de Porto y 12 de Estambul pensaban que la emergencia del trauma dental debe ser tratada inmediatamente. El 35,8\% de los profesores de Estambul y el $23 \%$ de los profesores de Porto tenían experiencia en trauma dental. El conocimiento de los medios óptimos de almacenaje para los dientes avulsionados permanentes fue especialmente pobre. En el presente estudio, la mayoría de los profesores no sabía la importancia del control de la vacuna tetánica en el trauma dental. (15)

En otro estudio realizado en el año 2003 realizaron una encuesta que consistía en siete preguntas simples con respecto a la avulsión dental que fue aplicada a 60 profesores de cinco escuelas primarias de Rió de Janeiro, Brasil para evaluar su conocimiento en el tema y establecer pautas a seguir cuando suceda un accidente de este tipo. Se observo una carencia de información técnica 
entre los profesores, la mayoría de ellos contestaron mas intuitivamente que sobre una base informativa. Este estudio demostró la necesidad de una comunicación más eficaz entre los profesionales y los profesores escolares para mejorar el manejo en las emergencias dentales. (16)

En el año 2006 se realizo una investigación referente el tema donde evaluaron el conocimiento sobre avulsión dental, para lo cual encuestaron a 117 profesores de primaria de Adamantina, São Paulo, Brasil. Los resultados muestran que el 75,2\% de profesores conocían la importancia del manejo de la emergencia y el 60,6 \% buscaría un dentista para el tratamiento de los casos; sólo el 18,8 \% reimplantaría el diente inmediatamente, el $55 \%$ lo lavarían con agua, el 42,7 \% lo mantendrían en un medio húmedo, pero sólo el 7,6 $\%$ lo mantendría en leche. El $33 \%$ de profesores ha tenido experiencia previa en avulsión dental. Este estudio muestra la falta de conocimiento de los profesores sobre avulsión y la necesidad de campañas educativas para mejorar el manejo de emergencia de la avulsión. (17)

De acuerdo a los datos presentados anteriormente y a que en nuestro país no contamos con datos referentes al nivel de conocimiento y conducta de los docentes sobre el traumatismo dentario, los resultados obtenidos en este estudio podrían incentivar campañas informativas en las escuelas sobre el manejo de un niño que sufre una avulsión o fractura dentaria; pues para que el tratamiento del odontólogo tenga un mejor pronóstico depende inicialmente de la conducta adecuada del docente frente a un caso de traumatismo dental.

\section{Objetivo general}

Evaluar el nivel de conocimiento y conducta de los docentes de Educación Inicial, docentes del 1er Ciclo, docentes del 2do Ciclo y docentes de Educación Física de las escuelas públicas de la ciudad de San Lorenzo (Paraguay) frente a casos de Avulsión y Fractura Dentaria durante el año 2009.

\section{Objetivos específicos}

Determinar el nivel de conocimiento y la conducta a seguir que tienen los docentes frente a un caso de Avulsión y Fractura Dentaria según género, edad, grado de capacitación, cargo y años de experiencia profesional.

Identificar la experiencia previa de los docentes frente con un caso de Avulsión y Fractura Dentaria según los años de experiencia profesional y al cargo que tienen; docentes de educación inicial, docentes del 1 ciclo, docentes del 2 ciclo y docentes de educación física.

\section{Material y métodos}

El diseño que se utilizo para este trabajo fue $\mathrm{Ob}-$ servacional, Descriptivo, de Corte Transverso.

Se incluyeron en el estudio 141 Docentes de Educación Inicial, Docentes de 1er ciclo, Docentes de 2 do ciclo y docentes de educación física de las escuelas públicas de la ciudad de San Lorenzo- Paraguay (Esc. Básica No 4196 Nuestra Señora de la Asunción Municipal No 8, Esc. Básica Nº 1881 Árabe Siria, Esc. Básica No 15340 “26 de Febrero La Victoria, Esc. Básica N 251 José Dolores González, Esc. Franklin Delano Rooseveelth, Esc. Básica Nº. 2393. Jorge A Gadea, Esc. Básica No 118 Coronel Luis Caminos, Esc. Básica No 5610 Nuestra Señora de la Paz, Esc. Básica $N^{o} 4183$ San Francisco de Asís Municipal No 5 , Esc. Básica No 4182 Virgen de Fátima, Esc. Básica No. 4184 Rufino Torales Municipal No 7, Esc. 
Básica N 599 Francisco Caballero Alvarez, Esc. Básica No 4185 San Isidro Labrador Municipal No 9, Esc.Básica No 598 Domingo Sávio).

El muestreo fue probabilístico por conglomerados en 2 etapas; pues los datos fueron tomados al azar de la lista de la Coordinación de Supervisiones Educativas zona 1 Departamento Central. El reclutamiento se solicito primeramente por medio de una carta de permiso a los Directores de las escuelas públicas de San Lorenzo donde se encontraban los docentes a encuestar sobre el conocimiento y conducta a seguir en el caso de una Avulsión y Fractura Dental. El levantamiento de datos estuvo a cargo de una Odontóloga calibrada para el estudio. Se tuvieron como criterios de inclusión: A los docentes que aceptaron participar del estudio, de 20 años en adelante, los docentes de Educación Inicial, Docentes de 1 ciclo, Docentes de 2 ciclo y docentes de educación física. Se excluyeron a los docentes que por algún motivo no se encontraban en la escuela en el momento de la encuesta; ya sea por enfermedad, luto, reposo, inclemencia del tiempo, etc.

Fue aplicado un cuestionario de 10 preguntas utilizando los siguientes intervalos para medir el conocimiento en: Alto (8-10 puntos), Moderado (5-7 puntos), Bajo (0-4 puntos) y para la conducta 10 preguntas utilizando los siguientes intervalos: Favorable (8-10 puntos), Regular (5-7 puntos), Desfavorable (0-4 puntos). Con las siguientes variables: Conocimiento, conducta, edad, sexo, grado de capacitación, cargo, Experiencia Profesional. En base a la escala del 60\% se consideraron las respuestas como correctas o incorrectas.

El tamaño mínimo de la muestra requerido fue de 138 con una proporción esperada de 0,10, una amplitud de 0,10 y un nivel de confianza del $95 \%$, se calculo en base a un estudio con 117 profesores (Morri 2007 (17)). El cálculo se realizo teniendo en cuenta el menor valor, empleando la tabla 6E para estudio descriptivo de una variable dicotómica del libro Diseño de investigaciones clínicas (18). Para el análisis de datos se utilizó estadística descriptiva.

En el estudio se cumplieron los criterios éticos para la investigación en salud, en cuanto a respeto, beneficio y justicia.

\section{Resultados}

El nivel de conocimiento sobre avulsión y fractura dentaria más frecuente de los docentes fue el moderado con un 50\% (70/141), seguido por el conocimiento bajo con un $27 \%(38 / 141)$ y por último el conocimiento alto con un 23\% (33/141) (gráfico 1).

La conducta a seguir sobre avulsión y fractura dentaria más frecuente por los docentes fue el de regular con un $47 \%$ (66/141), seguido por la conducta a seguir favorable con un 30\% (42/141) y por último la conducta a seguir desfavorable con un 23\% (33/141) (gráfico 2).

El nivel de conocimiento según el género indica que tanto el género masculino como el femenino

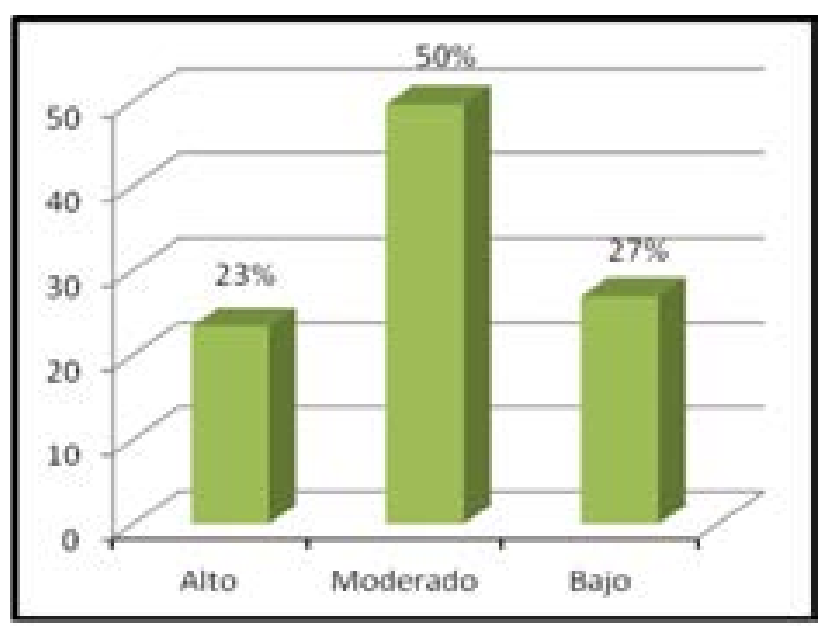

Grafico 1. Nivel de conocimiento en general de los docentes. $N=141$. 


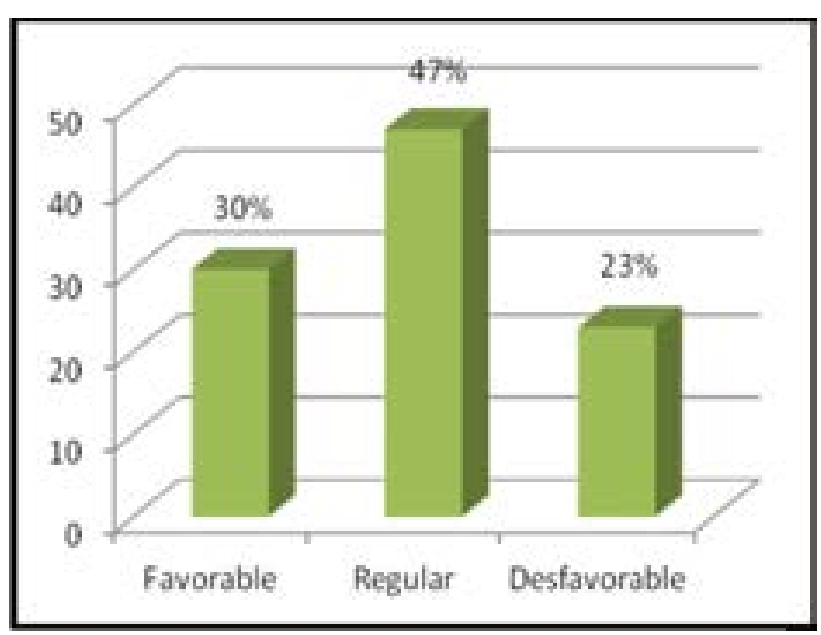

Grafico 2. Conducta a seguir sobre avulsión y fractura dentaria. $N=141$.

obtuvieron un conocimiento moderado, el sexo femenino con un $49,6 \%$, y el sexo masculino con un $50 \%$. La conducta a seguir según el género indica que tanto el género masculino como el femenino obtuvieron una conducta a seguir regular, el sexo femenino con un $44,7 \%$, y el sexo masculino con un $61,1 \%$.

El nivel de conocimiento según la edad los docentes indica que de 20 a 24 años de edad tienen un conocimiento bajo con un $60 \%$, los docentes de 25 a 44 años de edad tienen un conocimiento moderado, los docentes de 45 a más de 49 años de edad tienen un conocimiento alto. La conducta a seguir se presentó regular en todos los grupos de edad.

El nivel de conocimiento según el grado de capacitación indica que los docentes de educación inicial, los docentes de educación escolar básica, los docentes de educación física tienen un conocimiento moderado, los docentes con una carrera universitaria incompleta tienen un conocimiento alto con un 50\% (cuadro 1).

La conducta a seguir según el grado de capacitación indica que los docentes de educación inicial tienen un mismo porcentaje en conducta favorable, regular y desfavorable, los docentes de educación escolar básica tienen una conducta regular con un 49\% (51/104), los docentes de educación física tienen una conducta regular con un 54,5\% (6/11), los docentes con una carrera universitaria incompleta tienen un mismo porcentaje en conducta favorable y regular con un $37,5 \%(3 / 8)$.

El nivel de conocimiento según el grado de experiencia profesional indica que los docentes con menos de 1 año de experiencia profesional tienen un conocimiento bajo con un $100 \%$, los docentes con 1 a 5 años de experiencia profesional tienen un conocimiento moderado con un 61,1\%, los docentes con 6 a 10 años de experiencia profesional tienen un mismo porcentaje en conocimiento moderado y bajo con un $41,7 \%$, los docentes con 11 a 15 años de experiencia profesional tienen un conocimiento moderado con un $58,3 \%$, los docentes con 16 a 20 años de experiencia profesional tienen un conocimiento mo-

Cuadro 1. Nivel de conocimiento según grado de capacitación. N: 141.

\begin{tabular}{|c|c|c|c|c|c|c|c|c|}
\hline \multirow{3}{*}{ Edades } & \multicolumn{6}{|c|}{$\begin{array}{l}\text { Nivel de conocimiento se- } \\
\text { gún grado de capacitación }\end{array}$} & & \multirow{3}{*}{$\%$} \\
\hline & \multicolumn{2}{|c|}{ Alto } & \multicolumn{2}{|c|}{$\begin{array}{l}\text { Mode- } \\
\text { rado }\end{array}$} & \multicolumn{2}{|c|}{ Bajo } & & \\
\hline & $\mathbf{N}$ & $\%$ & $\mathbf{n}$ & $\%$ & $\mathbf{N}$ & $\%$ & & \\
\hline D.E.Inicial & 2 & 11,1 & 9 & 50,0 & 7 & 38,9 & 18 & 100 \\
\hline D.E.E.Basica & 26 & 25,0 & 52 & 50,0 & 26 & 25,0 & 104 & 100 \\
\hline D.E.Fisica & 1 & 9,1 & 6 & 54,5 & 4 & 36,4 & 11 & 100 \\
\hline U.Incompleta & 4 & 50,0 & 3 & 37,5 & 1 & 12,5 & 8 & 100 \\
\hline U.Completa & 0 & 0,0 & 0 & 0,0 & 0 & 0,0 & 0 & 0 \\
\hline Postgrado & 0 & 0,0 & 0 & 0,0 & 0 & 0,0 & 0 & 0 \\
\hline $\begin{array}{l}\text { Totales } \\
\text { (n: } 141)\end{array}$ & 33 & 23,4 & 70 & 49,6 & 38 & 27,0 & 141 & 100 \\
\hline
\end{tabular}


derado con un 47,1\%, los docentes con 21 a 25 años de experiencia profesional tienen un conocimiento alto con un $66,7 \%$. La conducta a seguir según años de experiencia profesional indica que los docentes con menos de 1 año de experiencia profesional tienen una conducta desfavorable con un $75 \%$, los docentes con 1 a 5 años de experiencia profesional tienen una conducta regular con un $41,7 \%$, los docentes con 6 a 10 años de experiencia profesional tienen una conducta regular con un 44,4\%, los docentes con 11 a 15 años de experiencia profesional tienen una conducta regular con un 50\%, los docentes con 16 a 20 años de experiencia profesional tienen una conducta regular con un $52,9 \%$, los docentes con 21 a 25 años de experiencia profesional tienen una conducta regular con un $58,3 \%$.

En cuanto a experiencia previa de traumatismo dental según años de experiencia profesional indica que los docentes con menos de 1 año de experiencia profesional no tuvieron ningún caso de traumatismo dental con ninguno de sus alumnos, el $91.7 \%$ de los docentes con 1 a 5 años de experiencia profesional no tuvieron casos de traumatismo dental con alguno de sus alumnos, $83,3 \%$ de los docentes con 6 a 10 años de experiencia profesional no tuvieron casos de traumatismo dental con alguno de sus alumnos, el 91,7\% de los docentes con 11 a 15 años de experiencia profesional no tuvieron casos de traumatismo dental con alguno de sus alumnos, el $82,4 \%$ de los docentes con 16 a 20 años de experiencia profesional no tuvieron casos de traumatismo dental con alguno de sus alumnos, el 66,7\% de los docentes con 21 a 25 años de experiencia profesional no tuvieron casos de traumatismo dental con alguno de sus alumnos. En total se puede observar que de los $141(100 \%)$ docentes encuestados el $(86,5 \%)$ no tuvieron casos de traumatismo dental con alguno de sus alumnos.
En cuanto a experiencia previa de traumatismo dental de acuerdo al cargo indica que el $100 \%$ de los docentes de E. Inicial no tuvieron casos de traumatismo dental con alguno de sus alumnos, el $86 \%$ de los docentes de 1er ciclo no tuvieron casos de traumatismo dental con alguno de sus alumnos, el $80 \%$ de los docentes de $2^{\circ}$ ciclo no tuvieron casos de traumatismo dental con alguno de sus alumnos, el 100\% de los docentes de E. Física no tuvieron casos de traumatismo dental con alguno de sus alumnos.

En cuanto a si recibió alguna información sobre traumatismo dental según años de experiencia profesional se encontró la mayoría de los docentes no recibieron ninguna información sobre traumatismo dental (Cuadro 2).

En el caso de un traumatismo dental los resultados indican que los docentes acudirían primero a un hospital o centro de salud con un 53,9\%. En

Cuadro 2. Recibió alguna información sobre traumatismo dentario.

\begin{tabular}{|c|c|c|c|c|c|c|}
\hline $\begin{array}{c}\text { Antigüedad } \\
\text { Si }\end{array}$ & $\%$ & No & $\%$ & Totales & \\
\hline $\begin{array}{c}\text { Menos de 1 } \\
\text { año }\end{array}$ & 0 & 0 & 4 & 100,0 & 4 & $100 \%$ \\
\hline $\begin{array}{c}\text { De 1 a 5 } \\
\text { años }\end{array}$ & 7 & 19,4 & 29 & 80,6 & 36 & $100 \%$ \\
\hline $\begin{array}{c}\text { De 6 a 10 } \\
\text { años }\end{array}$ & 3 & 8,3 & 33 & 91,7 & 36 & $100 \%$ \\
\hline $\begin{array}{c}\text { De 11 a } 15 \\
\text { años }\end{array}$ & 3 & 8,3 & 33 & 91,7 & 36 & $100 \%$ \\
\hline $\begin{array}{c}\text { De 16 a 20 } \\
\text { años }\end{array}$ & 5 & 29,4 & 12 & 70,6 & 17 & $100 \%$ \\
\hline $\begin{array}{c}\text { De 21 a } 25 \\
\text { años }\end{array}$ & 5 & 41,7 & 7 & 58,3 & 12 & $100 \%$ \\
\hline $\begin{array}{c}\text { Totales } \\
\text { (n: } 141) \\
(100 \%)\end{array}$ & 23 & 16,3 & 118 & 83,7 & 141 & $100 \%$ \\
\hline
\end{tabular}


Cuadro 3. En que trasportaría el diente avulsionado.

\begin{tabular}{|c|c|c|}
\hline $\begin{array}{c}\text { En que transportaría el diente } \\
\text { avulsionado. }\end{array}$ & $\mathbf{n .}$ & $\%$ \\
\hline En la boca del niño. & 2 & 1,4 \\
\hline En la mano del niño. & 6 & 4,3 \\
\hline En papel higiénico pañuelo o gasa & 57 & 40,4 \\
\hline En un vaso con agua & 28 & 19,9 \\
\hline En un vaso con leche & 23 & 16,3 \\
\hline En enjuague bucal & 13 & 9,2 \\
\hline No se & 12 & 8,5 \\
\hline Totales (n=141) (100\%) & 141 & 100 \\
\hline
\end{tabular}

el caso de una avulsión dental los resultados indican que los docentes llevarían el diente avulsionado al odontólogo $(84,4 \%)$.

En el caso de una avulsión dental los resultados indican que los docentes transportarían el diente avulsionado hasta la consulta odontológica en un papel higiénico, pañuelo o gasa con un 40,4\% (Cuadro 3).

\section{Discusión}

Aunque hoy en día los traumatismos dentales son la segunda causa de atención odontopediátrica tras la caries, en un futuro muy cercano la incidencia de las lesiones traumáticas constituirá la principal demanda de atención (1), por ello es importante saber que conocen los docentes sobre el tema ya que los niños se encuentran gran parte del día con ellos.

Los resultados obtenidos en el presente trabajo, indican que el nivel de conocimiento de los docentes sobre avulsión y fractura dentaria fue bajo en un $27 \%$, menor a la encontrada por SAELIM V que llego a la conclusión que el $63 \%$ de los docentes que encuesto no tenía conocimientos sobre el tema. (35)

En este trabajo se encontró que de los 141 docentes encuestados $118(83,6 \%)$ no recibieron información sobre traumatismo dental a lo largo de su carrera profesional, mayor a la encontrada por Blakytny, que llego a la conclusión que $66,1 \%$ (181 profesores de los 388 encuestados) no habían recibido ningún consejo sobre el manejo de la emergencia de un traumatismo dental. (20)

En cuanto a la experiencia previa en este trabajo indican que el 13,4\% de los docentes encuestados tuvieron experiencia previa en traumatismo dental, mayor a la encontrada en la investigación hecho por Mori GG donde el 33\% de los profesores ha tenido experiencia previa en avulsión traumatismo dental. (17)

En el caso que se presente una avulsión dental los resultados de este trabajo indicaron que el $84,40 \%$ de los docentes llevarían el diente avulsionado al odontólogo y que solo 6,38\% reimplantaría el diente inmediatamente, siendo según la literatura estudiada la primera opción de tratamiento de un diente avulsionado la reimplantación $(3,7,8$, $39,42)$, en la investigación hecho por Mori GG se encontraron prácticamente los mismos resultados, el 60,6\% buscaría un dentista para el tratamiento de los casos; sólo el 18,8\% reimplantaría el diente inmediatamente. (17)

En el caso que se presente una avulsión dental los resultados de este trabajo indicaron que el $40,43 \%$ de los docentes llevaría el diente avulsionado hasta la consulta odontológica en un papel higiénico, pañuelo o gasa, mayor a la investigación hecha por Pacheco, donde el $30 \%$ mantendrían el diente envuelto con papel tisú. (16)

De acuerdo a los resultados obtenidos varios son los investigadores que creen que importante 
incentivar a que se realicen campañas informativas en las escuelas sobre el manejo de un niño que sufre un traumatismo dental; pues para que el tratamiento del odontólogo sea exitosa depende básicamente de las decisiones que tomen los docentes si el niño se encuentra durante el accidente en la escuela. Solo con una buena información ellos tendrán una actitud adecuada frente a los traumatismos dentarios y por lo tanto el tratamiento realizado por el odontólogo tendrá un mejor pronóstico.

\section{Conclusión}

Los resultados obtenidos en este estudio indican que el nivel de conocimiento sobre traumatismo dental de los docentes fue moderado en un $50 \%$ de ellos, la conducta a seguir de los docentes en un $47 \%$ fue regular, sin diferencias de acuerdo al género, yendo en aumento de acuerdo a la edad, grado de capacitación y año de experiencia profesional. Hubo experiencia de traumatismo dental en la mayoría de los grupos y habían recibido poca información con respecto a traumatismos dentales.

En cuanto a un caso de un traumatismo dental los docentes acudirían primero a un hospital o centro de salud, llevarían el diente avulsionado al odontólogo en un papel higiénico, pañuelo o gasa.

Teniendo en cuenta los datos anteriormente presentados se torna necesario implementar campañas educativas sobre la atención de emergencias en Traumatismos Dentales en las escuelas, ya que en el ambiente escolar la persona más cercana al niño es el docente y su intervención en el momento que el niño sufre el traumatismo dental puede ser determinante para la resolución de los daños que éste pueda haber sufrido.

\section{Referencias}

1. García Ballesta Carlos-Pérez Lajarin Leonor. El Problema. Clasificación, etiología y patogenia. En: Traumatología Oral en Odontopediatría. Madrid. España. Editorial Ergon.2003. Pág. 11-33.

2. Del Castillo Pardo de Vera, J.L. Manual de Traumatología Facial. Traumatología Dentoalveolares. Editorial Ripano.1 ${ }^{a}$ Edición. Madrid, España. Año 2007.

3. Navarro Vila, Carlos. Tratado de Cirugía Oral y Maxilofacial. Traumatismos Dentarios. Editorial Aran Ediciones S. R. L. $1^{a}$ Edición. Madrid, España. Año 2004.Pág.491-513.

4. Basrani Enrique, Di Nallo Renee. Traumatología Dentaria en niños y adolescentes. El Paciente Traumatizado. Editorial

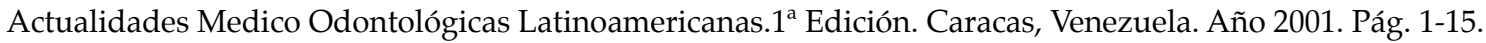

5. Cohen Stephen. Vías de la Pulpa. Traumatismos. Editorial Mosby. $8^{a}$ Edición. Barcelona. España. Año 2002. Pág. 595639.

6. Soares José - Goldberg Fernando. Endodoncia Técnica y Fundamentos. Lesiones Traumáticas de los tejidos dentarios. Editorial medica panamericana. Buenos Aires. Argentina. 2002 Pág.: 236-290.

7. Pinto Guedes Antonio Carlos y col. Rehabilitación Bucal en Odontopediatría. Tratamiento inmediato de las lesiones traumáticas en dientes permanentes jóvenes. Editorial Amolca. Caracas. Venezuela. 2003. Pág. 177-200.

8. Canalda Sahlí Carlos-Brau Aguade Esteban. Endodoncia Técnicas Clínicas y bases científicas. Traumatismos Dentales. Editorial Masson. Barcelona. España. 2001. Pág. 283-298.

9. Veleiro Rodriguez Carolina. Traumatismos Dentales en niños y adolescentes. Venezuela. 2001.

10. García Ballesta C, Pérez Lajarin Leo, Castejon Navas Isabel. Prevalecía y Etiología de los Traumatismos Dentales. Una Revisión: RCOE. Mar. Abri.2003.Vol. 8. N² 2.p 131-141 ISSN 1138-123X. 
11. Briñon Elida Norma. Lesiones e Iatrogénias en Odontología Legal. Editorial Ediciones Cátedra Jurídica. $1^{a}$ Edición Buenos Aires. Argentina Año 2006.Pág. 235-252.

12. Basrani Enrique. Blank Ana, Cañete M. Teresa. Radiología en Endodoncia. Análisis Radiográfico de la Patología Dentaria Adquirida en Relación con la Endodoncia. Editorial Actualidades Medico Odontológicas Latinoamericanas. $1^{\mathrm{a}}$ Edición. Caracas. Venezuela. Año 2003. Pág. 59-69.

13. Estrela Carlos, Ciencia Endódontica. Traumatismo Dentario. Editorial Artes Médicas Ltda.1 $1^{a}$ Edición. San Paulo, Brasil. Año 2005. Pág. 799-904.

14. Babeira Leache Elena, Boj Quesada Juan Ramón, Cátala Pizarro Montserrat, García Ballesta Carlos, Mendoza Mendoza Asunción. Odontopediatría. Lesiones Traumáticas de los Dientes en Desarrollo. Editorial Masson S.A. $2^{a}$ Edición. Barcelona. España. Año 2002. Pág.271-296.

15. Caglar E, Ferreira LP, Kargul B. Dental trauma management knowledge among a group of teachers in two south European cities. Dent Traumatol. 2005; 21(5):258-262.

16. Pacheco Lf, Filho PFG, Letra A, Menezes R, Villoria GEM, Ferreira SM. Evaluation of the knowledge of the treatment of avulsions in elementary school teachers in Rio de Janeiro, Brazil. Dent Traumatol. 2003; 19(2); 76-78.

17. Mori GG, Turcio KHL, BorroVPB, Maurisso, AM. Evaluation of the knowledge of tooth avulsion of school professionals from Adamantina, São Paulo, Brazil. Dental Traumatol. 2007; 23(1):2-5.

18. Hulley Stephen, Cummings Steven, Browner Warren, Grady Deborah, Newman Thomas. Diseño de investigaciones clínicas. Calculo de la potencia y del tamaño de muestra. Editorial ISBN. ${ }^{\circ}$ Edición. España. Año 2008. Pág. 73-105.

19. Da Cruz Campos Ma. Ines, Mota Henriques Karen Ayupe, Neiva Campos Celso. nivel de informacao sobre a conducta de urgencia frente ao traumatismo dental com avulsao. Pesq Bras Odontoped Clin Integr, Joao Pessoa, v. 6, n. 2, p.155159, mai./ago.2006.

20. Blakytny C, Surbuts C, Thomas A y Hunter ML. Avulsed permanent incisors: knowledge and attitudes of primary school teachers with regard to emergency management. International Journal of Paediatric Dentistry. 2001;11:327-332.

21. García Ballesta Carlos-Pérez Lajarin Leonor. Fracturas de la corona. En: Traumatología Oral en Odontopediatría. Madrid. España. Editorial Ergon.2003. Pág. 57-80.

22. Mendoza Mendoza Asunción. Fracturas de la raíz. En: Traumatología Oral en Odontopediatría. Madrid. España. Editorial Ergon.2003. Pág. 11-121.

23. Joaquín F López, Begoña García C, Silvia García V, Tratamiento de las lesiones dentales traumáticas, Acta Odontol. Venez.2006 v.44 n.3.

24. Panizza Eduardo, López Ma. del Carmen. Urgencias en Odontopediatría EN: Odontopediatría Hoy. MontevideoUruguay. 2002, p. 147-159.

25. Mendoza Mendoza Asunción. Fracturas coronoradiculares. En: Traumatología Oral en Odontopediatría. Madrid. España. Editorial Ergon.2003. Pág. 100-110.

26. Gallego Jesús, Martínez Roxana, Revista Latinoamericana de Ortodoncia y Odontopediatría. Traumatismos dentales en niños de 12-14 años en el municipio San José de las Lajas. La Habana. Cuba.

27. García Ballesta Carlos, Tratamiento de la avulsion. En: Traumatología Oral en Odontopediatría. Madrid. España. Editorial Ergon.2003. Pág. 164-177.

28. Traebert J. Almeida I.C.S. Garghetti C, Marcenes W.C. et al. Prevalência, necessidade de tratamento e fatores predisponentes do traumatismo na dentição permanente de escolares de 11 a 13 anos de idade. Cad . saúde Publica. Abr 2004. v. 20 n.2.

29. García Ballesta Carlos-Lesiones del ligamento periodontal. En: Traumatología Oral en Odontopediatría. Madrid. España. Editorial Ergon.2003. Pág. 122-143

30. Panzarini Sr, Pedrini D, Brandini Da, Poi Wr, Santos Mf, Correa Jpt, Silva Ff. Physical education undergraduates and dental trauma knowledge. Dent Traumatol. 2005;21(6):324-328.

31. García Ballesta Carlos-Pérez Lajarin Leonor, Lillo Cortes Olga. Lesiones de los tejidos blandos. En: Traumatología Oral en Odontopediatría. Madrid. España. Editorial Ergon.2003. Pág.45-55. 
32. Vanesa B, Boix H,Saez S, Ballet L. Traumatismos dentales en dentición permanente joven: A propósito de un caso. Rev Oper Dent Endod 2008; 5:84.

33. García Ballesta Carlos-Pérez Lajarin Leonor, López Nicolás M. Pautas de actuación en las lesiones traumáticas que cursan luxación. RCOE. 2003. v.8. n.2.

34. García Ballesta Carlos-Pérez Lajarin Leonor, Cozar Hidalgo A. Nuevas tendencias en el tratamiento de la avulsión dental. RCOE. 2003. v.8. n.2.

35. Sae-Lim V, Lim LP. Dental trauma management awareness of Singapore pre-school teachers. Dent Traumatol. 2001;17(2):71-76.

36. Mendoza Mendoza Asunción. Concusion y Subluxación. En: Traumatología Oral en Odontopediatría. Madrid. España. Editorial Ergon.2003. Pág.144-148.

37. De Deza Espasa Suarez, Galindo Marco, Poirier Aldeã, Farre Llort, Quesado Boj. Archivo de odontoestomatologia. junio 2001.v. 17 n. 5.

38. García Ballesta Carlos, Tratamiento de las luxaciones en la dentición permanente. En: Traumatología Oral en Odontopediatría. Madrid. España. Editorial Ergon.2003. Pág. 149-163.

39. Lllano de Andrés, Gómez Vicente, López Alberola. Avulsión dentaria o exarticulación en la infancia. Bol Pediatr 1995; 36: 333-335.

40. Andreasen Frances M. Traumatología dental y ortodoncia. Ortodoncia Clínica 2004;7 (1):8-20.

41. Ulver de Beluatti Valentina. Ferulización flexible en traumatología oral. Revista argentina de medicina y cirugía del trauma.2002 V 3. n.2.

42. Babeira Leache Elena, Boj Quesada Juan Ramón, Cátala Pizarro Montserrat, García Ballesta Carlos, Mendoza Mendoza Asunción. Odontopediatría. Lesiones Traumáticas del ligamento periodontal. Editorial Masson S.A. $2^{\text {a }}$ Edición. Barcelona. España. Año 2002. Pág.297-318.

43. Escobar Muñoz Fernando, Traumatismos en la dentición temporal en: Odontología Pediátrica, Caracas-Venezuela. Amolca.2004, p. 271-287.

Revisión y traducción de portugués: Fabián Calixto Fraiz

Recibido: 05-02-13

Aceptado: 25-06-2013

Correespondencia teresachirife@gmail.com 